\title{
Stress Prediction of Professional Students using Machine Learning
}

\author{
Disha Sharma, Sumit Chaudhary
}

\begin{abstract}
As the globalization, are expanding individuals twist towards the cutting-edge life Stress issue turns into a significant issue among in experts and understudies life. The term pressure is causing different mental issue face to face. Understudies of various courses and distinctive expert college are expanding ambushed with this pressure. The points of this examination are to research natural, social, mental and scholarly postgraduate and doctoral understudies. The quantity of tests of this investigation are 220 undergrad and postgraduate understudies. The information of my examination was gathered independent from anyone else - planned poll and by PSS Scale and the overview has organized inquiries which were gathered through google dox. There are many pressure expectation calculations has been proposed like SVM, KNN, RANDOM FOREST, NAVIE BAYES, LOGISTIC REGRESSION, DECISION TREE. Different machine learning methods are utilized that related in this field. Too, it talks about the application territories and difficulties for stress forecast with knowledge into the past research work.
\end{abstract}

Keywords: Stress, Prediction, classification, Machine learning, Questionnaires survey, weka tool.

\section{INTRODUCTION}

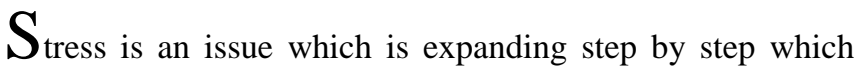
additionally influences the individual physical and mental wellbeing. [1] As the individual are moving toward the path for accomplishing development in their own and calling which is the significant reason for worry in the present life. Nowadays as the challenge is expanding in each field, the anxieties become the serious issue which influences the person's wellbeing which additionally causes genuine medical problems [2]. The expression "Stress" signifies when there is an issue which cause inconvenience to the individual and givethem any sort of mental issue. On the off chance that the pressure is antagonistic and influence the individual wellbeing is called misery and this additionally lead to the different mental issue or confusion. It sways on our body opposition which may cause may difficult issues which we are uninformed of and these sort of issue prompts serve physical medical problems like, coronary failure, mind related issue, rest issue [3]. Stress is basic in our day by day schedule life, which is pressure yet the vast majority of the pressure is trouble. Stress come when humans fails to wellbeing himself in the environment with high challenge. Mostly stress occurs when people tried to act against their emotion or mental condition [24].

Revised Manuscript Received on May 15, 2020.

* Correspondence Author

Disha Sharma*, Computer Science Engineering, Chandigarh University, Gharuan (Mohali), Punjab, India.

Dr. Sumit Chaudhary, Computer Science Engineering, Chandigarh University, Gharuan (Mohali), Punjab, India

(C) The Authors. Published by Blue Eyes Intelligence Engineering and Sciences Publication (BEIESP). This is an open access article under the CC BY-NC-ND license (http://creativecommons.org/licenses/by-nc-nd/4.0/)
Human body controls its inside condition by different physiological procedures, and keeps up at a specific condition of balance called as homeostasis [16].

"Hans Selye was the dad of stress specialists and have given numerous speculations on worry in look into. His view in 1956 was the "Stress is an inclination of enthusiastic or physical pressure." Selye accepted that the biochemical impacts of pressure would be experienced independent of whether the circumstance was certain or negative. In 2010, Hornby is characterized worry as "weight or stress achieved by issues for an amazing duration"[3, 2]. As per lazarus and Folkman in 1894, Stress emerges when an individual neglect to adapt to the extrenal condition or encompassing because of unexpected situation of exceptional requests on him/her. It rises when an individual has selected prerequisite (Fairbrother and caution, 2013) which consistently doesn't organize with his capacity [3]. In this time, understudies square measure casualties of economic process and its difficulties., family troubles, love disappointments (Blain and McArthur, 1961), negative economic, everyday problems (Carr and Umberson, 2013), and scholastic weight on the understudies square measure purpose of pressure currently a days in light-weight of the burden. apart from this displaying style, approach of mastering and affiliation among understudies and professional person within the study passageway square measure a component of distinct factors that deliver regarding strain $[23,15,3]$. throughout tests, When understudies neglects to urge nice stamps in assessment, they get American state persuaded that builds the suicidal inclination among them. consistent with United Nations agency reports in 2014, suicide worth is high amongst understudies because of the very fact of studious weight.[5, 3] In India ,857 understudies students complete it all because of the scholastic check pressure or weight, (Desai, 2006).[3] consistent with The National Crime Records Bureau a pair of,471 understudies have distributed suicide(2013) because of the very fact of assessment disappointment in India. to boot the days of India's Survey (September ten, 2014) has expressed that Martinmas of the understudies and seven.8 \% school understudies have endeavored suicide and out of every 3 suicide, there's one early life United Nations agency incorporates a location with the age gathering of 14-28 years. All things considered, "the subject of stress is bewildered and complex [13]. Stress can be defined by different aspects known as stressors including, social stress, environment, physiological, thoughts, emotional, anxiety [23][12].

\section{SOURCE OF STRESS}

We can experience stress from four basic sources:

Published By:

Blue Eyes Intelligence Engineering 


\section{Stress Prediction of Professional Students using Machine Learning}

\section{A. The Environment}

The external source of stress can occur by the environment in which the individual is unable to adjust with the external or internal stimulus which result in the cause of stress. Examples of environmental or external stressors include weather - cold or hot, disturbance, crowding, pollution, traffic, unsafe environment and increase level of crime [23].

\section{B. Social Stress}

The outside wellspring of stress can happen by the earth wherein the individual can't modify with the outer or inward improvement which bring about the reason for pressure. Instances of natural or outside stressors incorporate climate - cold or hot, unsettling influence, swarming, contamination, traffic, hazardous condition and increment level of wrongdoing.

\section{Physiological}

Everybody can encounter various Stressors throughout their life, on account of the distinctive social job we play in our public activity. Each individual assumes an alternate social job in their everyday life so as to live in a general public, for example, parent, life partner, guardian and representative. A few instances of social stressors incorporate cut-off times, money related issues, work uncertainty, introduction, contradictions, interest for your time and consideration, loss of cherished one, separate, coworking. This pressure happens when an individual can't assume a social job and not ready to rival the various individuals from the general public.

\section{Thoughts}

Each individual is unique and each individual see a similar circumstance in an alternate sense or way. Some individual translates and sees circumstance as upsetting, troublesome, agonizing and some individual see the conditions as a lovely and attempt to unravel that circumstance smoothly and quietly. Some circumstance in life are pressure inciting, however it is our considerations that decide if they are issue for us and open door for us.

Machine learning has huge application in every area and it is growing rapidly because of its effective [4] approaches in prediction and classification. The dataset used in this approach. Basically, machine learning are two types supervised and Unsupervised.[1] Now we are discussing some techniques of machine learning are -:

\section{A. Naïve Baye's}

Naive Baye's classifier belongs to the formula of probabilistic classifier [1]. It is based on the Baye's Theorem. Despite its naive design and simplicity i.e. $\mathrm{t}$ can be applied to very complex problems. This classifier is based on the assumption that feature is independent of any other feature. Naive Baye's classifier uses maximum label time.

\section{B. Logistic Regression}

Logistic Regression is a type of technique used in machine learning. Logistic regression is widely used for the statistical model. Logistical regression is also known as statistical learning technique in supervised machine learning method dedicated to 'classification' task.

\section{MACHINE LEARNINGTECHNIQUES}

\section{Multilayer Perceptron}

Multilayer perceptron (MLP) is a class of feed forward neural system. It comprise of three layers. They are related with different loads. The Input layer ,Hidden Layer and the Output Layer [4].

\section{Baye's Net}

Baye's Net utilizes the strategy that can actuate a Bayesian system $B$, that encodes a circulation $\mathrm{PB}(\mathrm{A} 1, \ldots, \mathrm{An}$, C), from a given preparing set.

We would then be able to utilize the subsequent display so given a lot of characteristics a1,...,an, the classifier dependent on $\mathrm{B}$ restores the name $\mathrm{c}$ that boosts the back likelihood $\mathrm{PB}(\mathrm{c} \mid \mathrm{a} 1, \ldots, \mathrm{an})$.

\section{LITERATURE REVIEW}

Researchers have discovered that the uses of machine learning algorithm for stress prediction by forming the patterns of questionaries dataset and another dataset.

U. Reddy et al. [1] Stress issue is a typical issue for the individuals those are working in IT proficient industry. Individuals working in that industry, face issue like change in their way of life and work societies, which has overstated the danger of stress. As per their paper they utilizing OSMI(open sourcing Mental Illness) Survey dataset 2017 from tech industry.[1] Different Machine learning strategies utilized (boosting, packing, Decision trees, Random timberland) and various kinds of characteristics they utilizing like sexual orientation, age, family ancestry eproduce medical advantages and so on. As per study they found that $75 \%$ individuals working in tech organization were marginally at the danger of creating pressure [1].

G. Harrison et al. [2] In the realm of globalization, the innovation is expanding quickly and we as a whole encompass by advancements which is valuable in our everyday life and one of the most fundamental innovation is cell phone which become the significant piece of everyone's life. As indicated by Occom's 2017 records, 94\% of grownups in UK have a cell phone; and more than three fourth of those are advanced mobile phones [2]. Deloitte's portable purchaser study (2016) proposes 33\% of Smartphone clients don't really makes voice calls by any stretch of the imagination. Rather, our telephones are utilized as portable PCs, for browsing email, shopping internet, getting to news, downloading music and recordings, taking part in web-based social networking, requesting nourishment, taking a gander at maps... the rundown goes on. We rely upon the web for each solution to our inquiries. on the We are reliant on innovation or simply dependent on this. We feel truly awkward and fragmented when we overlooked our cell phone [2]. Truth be told, as indicated by inquire about has demonstrated that a couple of individuals experience basic weight and uneasiness when they are disengaged from their phones.

Samrat Qaraqe et al. [3] As indicated by this paper plainly these sensor sign can distinguish feeling of anxiety where as physiological detecting gadgets are utilized to gather these signs. Signal pre-processing must be executed to select valuable highlights from accumulated sign. To choose valuable highlights from the accumulated sign, signal prehandling must be actualized [3].

Published By:

Blue Eyes Intelligence Engineering 
When the highlights are fearless, the AI calculations can be applied to assemble the grouping model. The accelerometer information can identify development and furthermore considered as measure to see physical exercises. Pulse varieties, galvanic skin reaction and skin temperature can reflect self-sufficient sensory system movement so their highlights are extremely helpful for anticipating the feeling of anxiety of person. Bolster vector machine, choice trees and Random woods are instances of powerful order calculations for mental pressure identification. Besides warm imaging innovation just as wearable sensors can be utilized for discovery. Once of the promising procedures o foresee singular feeling of anxiety is profound learning.

Huijie Lin et al. [4]. As indicated by report youngsters somewhere in the range of 8 and 11 years, will confront different psychosocial stressors, they revealed that understudy with lower monetary status, who get cash from their companions, made clash among relatives and furthermore lead a worry toward understudies. Stress was found to massively to influence understudies' wellbeing and physical movement including lack of sleep, weakness, and uneasiness which cause low fixation and lead to sorrow. Sandhi and Asrabadi (1994). In an investigation in the midst of college understudies announced achiness to visit the family, racial segregation, language hindrances, as the primary driver of worry among understudies. Understudies is likewise discovered hard to settle in another workplace uniquely who are away from home and they observe nature as a factor of pressure. Due to the non accessibility of darlings and absence of understudies embraces the outrageous degree of stress. As the understudies originate from different social foundations so they feel isolated from their way of life and feeling have them a culture stun. They stay tangled between odd estimations of their way of life and new standards where they are living, which gets clashes and uneasiness them. Additionally, verbal and nonverbal correspondence, methods of conduct contrast from culture to culture likewise turn into the explanation of worry in understudies [17]. Subsequently, correspondence issue in new condition was likewise detailed in their investigation which lead to worry among the understudies.

N. C. F. Codella et al. [5] proposed novel deep learning ensembles for melanoma recognition in dermoscopy images. They have proposed a system for the segmentation and classification of melanoma from dermoscopic images of skin. The method was evaluated on the largest public benchmark for melanoma recognition available. The proposed work is only applicable for linear image during the classification process and need to improvement in the pre-processing steps for further usages of non linear images.

Chigerwe, Boudreaux et al. [6] utilized the Maslach Burnout Inventory-Educator Survey (MBI-ES) on veterinary therapeutic understudies. To the creators' information, it had recently been led on therapeutic understudies, yet not veterinary restorative understudies (Chigerwe et al., 2014). The destinations of this examination were to assess utilization of the MBI-ES on veterinary understudies so as to assess the degree of stress experienced by the understudies every semester (Chigerwe et al., 2014). The MBI-ES assesses burnout, including passionate weariness, depersonalization, and low close to home achievement. Chigerwe et al. (2014) discovered "proof exists supporting that passionate pressure is common in veterinary restorative understudies. In this way, examination of the ideal device to evaluate the degree of burnout will aid the assurance and distribution of understudy bolster assets".

W.Smit et al. [7]. Biobeats to perceive pressure plans using existing sensors in mobile phones and wearable contraptions. With money related pros including Will Smith and Deepak Chopra, BioBeats is enabling individuals to make safety measure move against mental shakiness. Using wearable sensors joined with an application, similarly as an AI system in the cloud to perceive, check and treat mental issue. The purpose of there is to perceive how their body and cerebrum responds to weight and how weight impacts their own and master life [6].

\section{PERFORMANCE PARAMETERS}

\section{A. Kappa Statistic}

This is used to measure the performance of the machine learning technique[1]. It is a measure to test the interrater. Cohen's kappa is always less than or equal to 1. Formula can be used-:

$$
\begin{aligned}
& K=\text { Kappa statistics } \\
& P(A)=\text { percentage of agreement } \\
& P(E)=\text { chances of agreement }
\end{aligned}
$$

$$
\mathrm{K}=\mathrm{P}(\mathrm{A})-\mathrm{P}(\mathrm{E}) \backslash 1-\mathrm{P}(\mathrm{E})
$$

\section{B. True Positive Rate(TPR)}

It measures the values which are correctly classified as true i.e. belonging to a class .

\section{False Positive Rate(FPR)}

It measures the values which are incorrectly classified as true i.e. belonging to a class.

\section{Precision}

It processes the percentage of records that the classifier categorized as helpful are actually positive.

$$
\text { precision }=\mathrm{TP} / \mathrm{TP}+\mathrm{FP}
$$

\section{E. Recall}

Generally, we can use recall for completeness what percentage of positive tuple did the classifier label as positive. In recall Perfect Score is 1.0. In simple Words we can say that Inverse relationship between Precision and Recall

$$
\text { Recall }=\mathrm{TP} / \mathrm{TP}+\mathrm{FN}
$$

\section{F. F-Score}

It is calculated by finding out the harmonic mean [5] of precision and recall.

Precision $=$ Two $\mathrm{x}$ Precision $\mathrm{x}$ Recall $/$ Precision + Recall.

\section{G. MCC}

In machine learning this coefficient is utilized to build up the nature of the classifier utilized for the grouping in the model. It is determined by mulling over the True positive, False positive, True Negative and False Negative qualities. It generally gives the incentive between -1 and +1 [6]

Published By:

Blue Eyes Intelligence Engineering

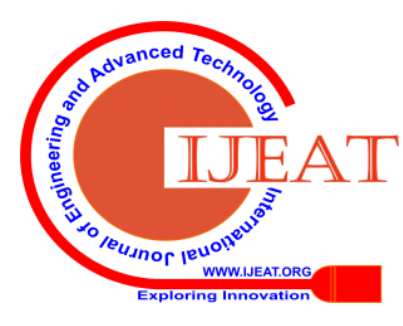




\section{Stress Prediction of Professional Students using Machine Learning}

\section{DATA SET AND PREPROCESSING}

The dataset was collected for PSS test which includes 14 questions overall including the entire emotional [9] question. The marking for the questions was in 5 ways (a) Never- 0 (b) Almost Never-1 (c) Sometimes -2 (d) fairly Often-3 (e) Very Often - 4. The stress level divided into three types Low, Moderate, high. The dataset was gathered from graduate and post - graduate students in the university. Through survey we are asked basic questions about their feelings in situations that they might have encountered in the last month and their reactions to it. Their answers are given some amount of weights and the weights thus help to calculate a score to analyze the stress level of the individuals. The dataset was pre-processed to analyze the weights and calculate final scores.

PSS (perceived stress scale) -: The Perceived Stress Scale (PSS) is a great stress evaluation instrument. This was founded by a physiological research Sheldon Cohen in 1983. [7] The inquiries in this scale pose about your sentiments , emotions , feeling and considerations during the past few month and present.

\section{PROPOSED APPROACH}

In this paper, we are using different types of Machine Learning technique to predict the stress level of the students. In the questionaries test, we understand the student's different types circumstances and situations. This proposed model includes PSS dataset collection, pre-processing, feature extraction and below figure (2) shows the comparison on the basis of their performance parameter.

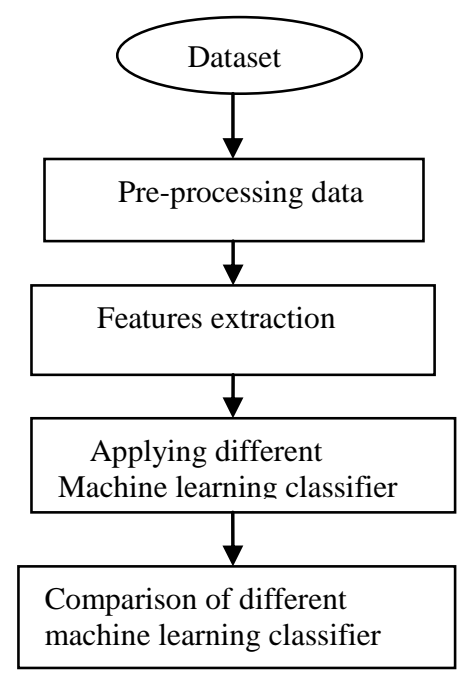

Fig-1(overall methodology)

\section{EXPERIMENTAL RESULT}

In this paper we can used own generated stress data set from survey. Stress data set contains 220 instances which have 6 attributes.

The data set has:

Number of Instances $=220$

Number of Attributes $=6$

All data set are visualized in figure 1.

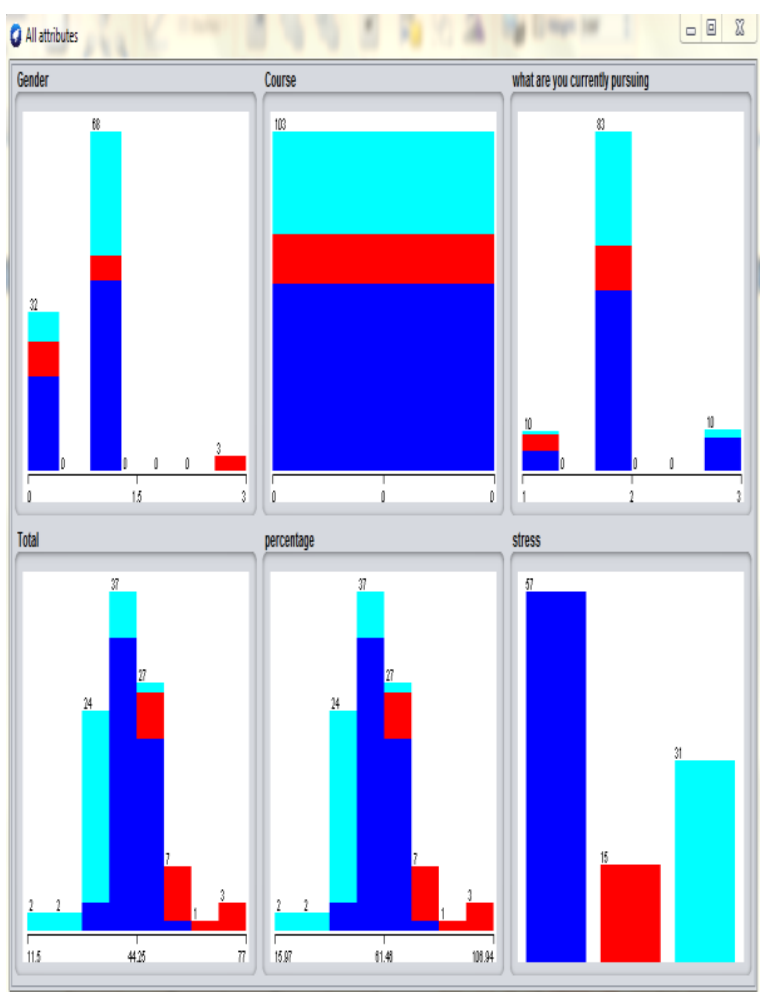

Fig -2(visualized data)

Table-I: We classified correctly and incorrectly instances.

\begin{tabular}{|l|l|l|l|l|}
\hline Classifier & $\begin{array}{l}\text { Correctly } \\
\text { instances }\end{array}$ & $\begin{array}{l}\text { Incorrectly } \\
\text { Instances }\end{array}$ & $\begin{array}{l}\text { Kappa } \\
\text { Statistic }\end{array}$ & \\
\hline Baye's Net & $88.3495 \%$ & $11.6505 \%$ & 0.7969 & \\
\hline $\begin{array}{l}\text { Multilayer } \\
\text { Perceptron }\end{array}$ & $85.4369 \%$ & $14.503 \%$ & 0.7444 & \\
\hline Naïve baye's & $86.4078 \%$ & $13.5922 \%$ & 0.7612 & \\
\hline $\begin{array}{l}\text { Logistic } \\
\text { Regression }\end{array}$ & $84.466 \%$ & $15.534 \%$ & 0.7308 & \\
\hline
\end{tabular}

Table-II: We have value of Mean Absolute error (MAE), Root Mean Square Error(RMSE), Relative Absolute Error.

\begin{tabular}{|l|l|l|l|}
\hline Classify & MAE & RMSE & RAE \\
\hline Baye's Net & 0.92 & 0.2741 & 23.4989 \\
\hline $\begin{array}{l}\text { Multilayer } \\
\text { perceptron }\end{array}$ & 0.125 & 0.2657 & 31.99 \\
\hline $\begin{array}{l}\text { Naive Baye's } \\
\text { Logistic } \\
\text { Regression }\end{array}$ & 0.129 & 0.283 & 33.047 \\
\hline
\end{tabular}

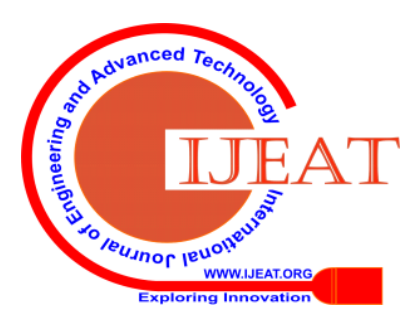


Table-III: We have value of TP (true positive), FP (false positive), precision, Recall.

\begin{tabular}{|l|l|l|l|l|}
\hline Classify & TP & FP & precision & Recall \\
\hline $\begin{array}{l}\text { Baye's } \\
\text { Net }\end{array}$ & 0.883 & 0.110 & 0.884 & 0.883 \\
\hline $\begin{array}{l}\text { Multilayer } \\
\text { Perceptron }\end{array}$ & 0.564 & 0.139 & 0.855 & 0.854 \\
\hline $\begin{array}{l}\text { Naive } \\
\text { Baye's }\end{array}$ & 0.864 & 0.127 & 0.8666 & 0.864 \\
\hline $\begin{array}{l}\text { Logistic } \\
\text { Regression }\end{array}$ & 0.845 & 0.132 & 0.844 & 0.844 \\
\hline
\end{tabular}

Table-IV: We have value of F-score, MCC, ROC Area.

\begin{tabular}{|l|l|l|l|}
\hline Classifier & F-Score & MCC & Area \\
\hline Baye's Net & 0.881 & 0.786 & 0.824 \\
\hline $\begin{array}{l}\text { Multilayer } \\
\text { Perceptron }\end{array}$ & 0.852 & 0.733 & 0.890 \\
\hline Naive Bayes & 0.863 & 0.754 & 0.901 \\
\hline $\begin{array}{l}\text { Logistic } \\
\text { Regression }\end{array}$ & 0.844 & 0.718 & 0.907 \\
\hline
\end{tabular}

With the help of classifier, we can make graph.

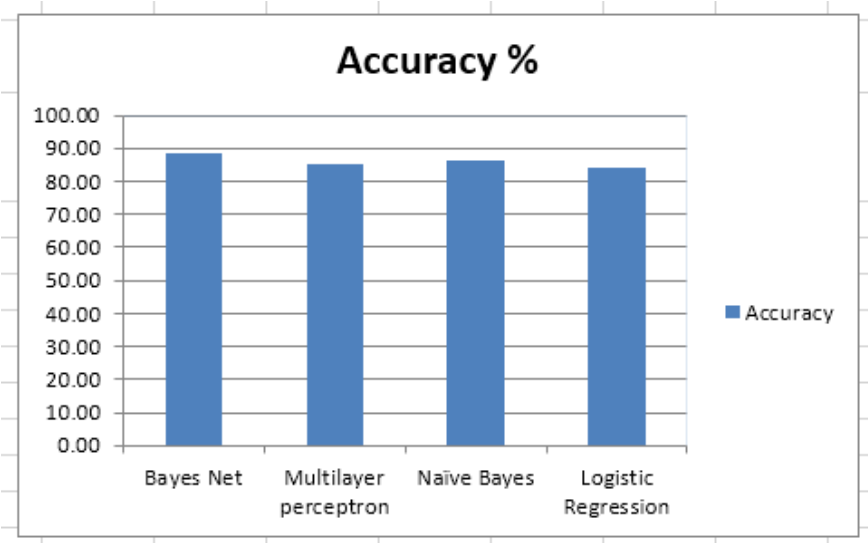

(Fig: 3 Graph)

\section{CONCLUSION}

Stress is a problem which is increasing day by day which also affects the individual physical and psychological health. The classification techniques we have used Naive Baye's, Baye's Net, Logistic Regression, Multilayer perceptron technologies for the prediction. Using Weka tool, the accuracy of various techniques is calculated and compared. According to this paper we observe the Kappa, statistic Fmeasure, MCC, mean absolute error, ROC area, false positive, true positive, RMSE and Recall Baye's Net classifier gives the longest accuracy of 88 percentage.

\section{REFERENCES}

1. U Srinivasulu Reddy, Aditya vivek Thota, Adharun," Machine learning Techniques for Stress prediction in working employee" 2018 IEEE International Conference on computational intelligence and computing research.

2. G. Mikelsons, M. Smith, A. Mehrotra, M. Musolesi, "Towards deep learning models for psychological state prediction using smartphone data: Challenges and opportunities, 2017.

3. Samrat Bisai, Richa Chaudhary, "Stress Among the Students of an Engineering Institution in India: An Empirical Analysis", Jindal Journal of Business Research, Volume: 6 issue: 2, page(s): 186-198 Article first published online: October 30, 2017; Issue published: December 1, 2017

4. Huijie Lin, Jia Jia, Quan Guo, Yuanyuan Xue, Qi Li, Jie Huang, Lianhong Cai, and Ling Feng, "User-level psychological stress detection from social media using deep neural network", 2014 In Proceedings of the 22nd ACM international conference on Multimedia (MM '14). ACM, New York, NY, USA, 507-516

5. C. Liao, R. Chen, and S. Tai, Emotion stress detection using EEG signal and deep learning technologies - IEEE Conference Publication, 2018 IEEE Int. Conf. Appl. Syst. Invent., no. 2, pp. 9093, 2018

6. Chigerwe et al. BMC Medical Education 2014, Published on: 28 November 2014

7. https://www.forbes.com/sites/lucysherriff/2019/ 03/25/how-onecompany-is-using-ai-to-address-mental-health

8. V. J. Madhuri, M. R. Mohan, R. Kaavya, "Stress Management Using Artificial Intelligence", 2013 Third International Conference on Advances in Computing and Communications, pp. 54-57, 2013.

9. https://das.nh.gov/wellness/Docs/Percieved\%20Stress\%20Scale.pdf

10. Siddhesh Keshkamat1, Shivani Kelkar2, Ashlesha Mhatre3, Sanjeev Dwivedi4," Survey: Stress Management Using Artificial Intelligence", International Advanced Research Journal in Science, Engineering and Technology Vol. 3, Issue 3, March 2016

11. https://www.open.edu/openlearn/health-sports-psychology/mentalhealth/managing-stress-and-anxiety-the-digital-age-the-dark-sidetechnology

12. R. Wang, G. Harari, P. Hao, X. Zhou, and A. T. Campbell SmartGPA: how smartphones can assess and predict academic performance of college students. In UbiComp'15, 2015.

13. Y. Suhara, Y. Xu, and A. Pentland. Deepmood: Forecasting depressed mood based on selfreported histories via recurrent neural networks. In WWW'17, April 2017.

14. S. Servia-Rodríguez, K. K. Rachuri, C. Mascolo, P. J. Rentfrow, N Lathia, and G. M. Sandstrom. Mobile sensing at the service of mental well-being: a large-scale longitudinal study. In WWW'17, April 2017.

15. Calaguas, G.M. "Survey of college academic stressors: Development of a new measure", International Journal of Human science", Volume: 9 Issue: 1 Year: 2012

16. S. Servia-Rodríguez, K. K. Rachuri, C. Mascolo, P. J. Rentfrow, N. Lathia, and G. M. Sandstrom. Mobile sensing at the service of mental well-being: a large-scale longitudinal study. In WWW'17, April 2017.

17. V. Pejovic, A. Mehrotra, and M. Musolesi. Anticipation mobile digita health: Towards personalized proactive therapies and prevention strategies. In Anticipation and Medicine, pages 253-267. Springer, 2017.

18. Norizam, Sulaiman. Determination and classification of human stress index using nonparametric analysis of EEG signals. Diss. Universit Teknologi MARA, 2015

19. Selvaraj, N. Psychological acute stress measurement using a wireless adhesive biosensor. In: 2015 37th Annual International Conference of the IEEE Engineering in Medicine and Biology Society (EMBC). 2015, p. 3137-3140.

20. Subhani, Ahmad Rauf, Wajid Mumtaz, Mohamed Naufal Bin Mohamed Saad, Nidal Kamel, and Aamir Saeed Malik. "Machine learning framework for the detection of mental stress at multiple levels." IEEE Access 5 (2017): 13545-13556.

Published By:

Blue Eyes Intelligence Engineering

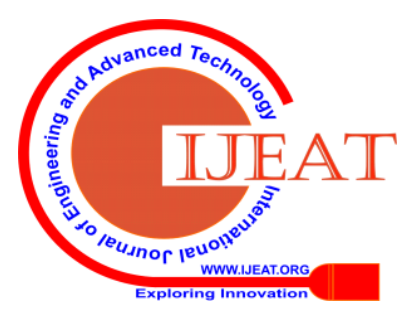


21. Adnan, Nadia, et al. "University students stress level and brainwave balancing index: Comparison between early and end of study semester." Research and Development (SCOReD), 2012 IEEE Student Conference on. IEEE, 2012.

22. Liu, David, and Mark Ulrich. "Listen to Your Heart: Stress Prediction Using Consumer Heart Rate Sensors." Online] 2014.

23. Selvaraj, N. Psychological acute stress measurement using a wireless adhesive biosensor. In: 2015 37th Annual International Conference of the IEEE Engineering in Medicine and Biology Society (EMBC). 2015, p. 3137-3140.

24. N. H. A. Hamid, N. Sulaiman, S. A. M. Aris, Z. H. Murat, and M. N. Taib, "Evaluation of human stress using EEG Power Spectrum," in 2010 6th International Colloquium on Signal Processing \& Its Applications (CSPA), Mallaca City, 2010, pp. 1-4

\section{AUTHORS PROFILE}

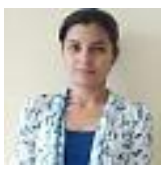

Disha Sharma is a M.E. Research Scholar at Department of C.S.E., University Institute of Engineering, Chandigarh University (CU), Gharuan (Mohali), Punjab, India.

She received Her B. Tech degree in Computer Science Engineering from kurukshetra University, India. Her interest includes Machine learning, Cloud computing, Artificial Intelligence.

Dr. Sumit Chaudhary is Associate Professor in Computer Science \& Engineering Department, at Chandigarh University, Gharuan (Mohali) Punjab, India. He has done Ph.D. degree in Computer Science \& Engineering from Uttaranchal University, Dehradun (Uttarakhand) in 2018. His area of research includes Big data Analytics, Cloud Computing, Wireless sensor network, Network Security, artificial Intelligence, MANET (Mobile Ad-Hoc network and Machine Learning.

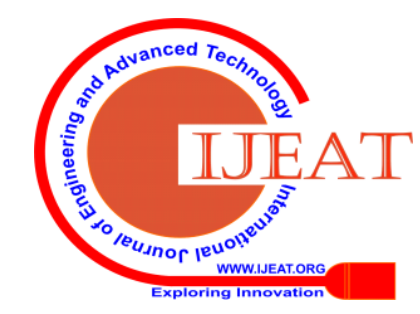

